\title{
Can the relic density of self-interacting dark matter be due to annihilations into Standard Model particles?
}

\author{
Xiaoyong Chu, ${ }^{a}$ Camilo Garcia-Cely ${ }^{b}$ and Thomas Hambye ${ }^{b}$ \\ ${ }^{a}$ International Centre for Theoretical Physics, ICTP, \\ Strada Costiera 11, Trieste, 34014 Italy \\ ${ }^{b}$ Service de Physique Théorique, Université Libre de Bruxelles, \\ Boulevard du Triomphe, CP225, Brussels, 1050 Belgium \\ E-mail: xchu@ictp.it, Camilo.Alfredo.Garcia.Cely@ulb.ac.be, \\ thambye@ulb.ac.be
}

ABSTRACT: Motivated by the hypothesis that dark matter self-interactions provide a solution to the small-scale structure formation problems, we investigate the possibilities that the relic density of a self-interacting dark matter candidate can proceed from the thermal freeze-out of annihilations into Standard Model particles. We find that scalar and Majorana dark matter in the mass range of $10-500 \mathrm{MeV}$, coupled to a slightly heavier massive gauge boson, are the only possible candidates in agreement with multiple current experimental constraints. Here dark matter annihilations take place at a much slower rate than the self-interactions simply because the interaction connecting the Standard Model and the dark matter sectors is small. We also discuss prospects of establishing or excluding these two scenarios in future experiments.

KeYwords: Beyond Standard Model, Cosmology of Theories beyond the SM

ARXIV EPRINT: 1609.00399 


\section{Contents}

\section{Introduction 1}

2 Four portals for SIDM annihilations into SM particles 2

2.1 Basic requirements 2

2.2 Four portals to the SM 4

$\begin{array}{lll}2.3 & \text { Surpassing indirect detection constraints } & 6\end{array}$

3 Scenarios with a $Z^{\prime}$ boson $\quad 7$

3.1 Majorana dark matter 8

$\begin{array}{lll}3.2 & \text { Scalar dark matter } & 12\end{array}$

4 Conclusions $\quad 14$

\section{Introduction}

Observations of dynamics of galaxies, clusters of galaxies and the Universe at large scales strongly support the Cold Dark Matter (CDM) paradigm. This suggests that most of the matter of the Universe consists of non-relativistic collisionless particles not present in the Standard Model (SM) of particle physics. In spite of these successes, within the CDM paradigm, a number of difficulties - such as the too-big-to-fail $[1,2]$ and the core-vscusp $[3,4]$ problems - have been found in N-body simulations of formation of small-scale structures, most notably of dwarf and low-surface-brightness galaxies. For a review of these problems, see ref. [5].

Strongly self-interacting dark matter (SIDM) is a plausible solution to some of these challenges [6]. Its key ingredient is the hypothesis that dark matter (DM) particles scatter off each other in small-scale structures with a cross section per unit of mass of around $1 \mathrm{~cm}^{2} / \mathrm{g}$ [7-14]. This corresponds to $10^{12} \mathrm{pb}$ for DM masses around $1 \mathrm{GeV}$, which is orders of magnitude above the standard thermal freeze-out cross section of about $1 \mathrm{pb}$; for a comprehensive discussion of alternative production regimes in the context of SIDM, see ref. [15]. Clearly, if DM undergoes a thermal freeze-out in the early Universe, some mechanism should be at work in order to explain this disparity of cross sections.

Two of these mechanisms have been discussed extensively in the literature. One of them is invoking a light mediator enhancing DM self-interactions via non-perturbative effects in small-scale structures [16-19]. The other one is considering DM annihilation processes in the early Universe that are induced by a relatively strong interaction but that are nevertheless phase-space suppressed due to the presence of many particles in their initial state $[20,21]$. In both cases, the production of DM proceeds via annihilations within the hidden sector. In the first case, DM annihilates dominantly into the light mediator 
responsible for the self-interactions. In the second case, three or four [22] DM particles annihilate into two of them. Thus in both scenarios an interaction connecting the DM and the SM sectors is not necessary for the DM self-interactions or annihilations. ${ }^{1}$ Needless to say, without such an interaction we will never discover the DM particle and will only be able to probe it through its gravitational and/or its self-interaction effects.

Although it is possible that after inflation no connector between both sectors has played any major role for DM annihilation and self-interaction processes, in this work we explore a third mechanism, largely overlooked to the best of our knowledge, in which the relic density of SIDM stems from the freeze-out of its annihilations into SM particles. In other words, we will show that DM self-interacting in a hidden sector must not necessarily annihilate into particles beyond the SM.

If, as we will assume all along this work, the large self-interaction cross section does not result from non-perturbative effects associated to the exchange of a lighter mediator, the DM particle must lie below the GeV scale. Searches of particles beyond the SM severely constrain such scenarios, basically restricting sub-GeV DM to be a singlet under the SM gauge group and requiring it to have rather small interactions with the SM particles. This is the mechanism we explore in this work: even though the DM sector has relatively strong interactions, its portal to SM particles - which are the dominant annihilation products of DM - is comparatively small and leads to a thermal freeze-out in agreement with the observed abundance of DM.

This article is organized as follows. We start off in section 2 by determining the possible scenarios giving rise to annihilations of sub-GeV DM into SM particles, based on the four possible portal interactions that are allowed by SM symmetries. From this discussion, only one scenario emerges, which is based on the portals that include an extra gauge boson. In section 3, we discuss such scenario in detail and examine the long list of corresponding experimental and observational constraints. Possibilities of future particle physics tests, associated to the fact that DM annihilates into SM particles, are also analyzed. Finally, we present our conclusions in section 4 .

\section{Four portals for SIDM annihilations into SM particles}

\subsection{Basic requirements}

In order that SIDM annihilates dominantly into SM particles, there is a number of preliminary basic requirements that it must fulfill. These are:

\section{- The DM mass must be below the GeV scale.}

As already mentioned above, we do not consider the possibility of a mediator with mass much smaller than the DM mass $m_{\mathrm{DM}}$, inducing large DM self-interactions through non-perturbative effects. This is because, if the mediator is a particle beyond the SM, such an option would easily allow the DM particles to annihilate dominantly

\footnotetext{
${ }^{1}$ Such an interaction might be nevertheless necessary for other concerns. For instance, for inducing the decay of the mediator in order to satisfy BBN and CMB constraints [23] or for establishing kinetic equilibrium between DM and SM thermal bath in the 3-to-2 framework [21, 24, 25].
} 
into a pair of mediators, rather than into SM particles. If instead the mediator inducing non-perturbative effects is a SM particle such as a photon or a massive boson, a sufficiently large self-interaction cross section could hardly be accommodated without violating experimental constraints [19, 26, 27].

In absence of lighter mediators, provided the associated dark sector couplings, $g_{D}$, have perturbative values, the self-interaction cross section can be calculated by means of the ordinary Born expansion in the small-velocity limit [19]. In this case, for a self-interaction induced by the exchange of a mediator with mass of order $m_{\mathrm{DM}}$, dimensional analysis shows that $\sigma_{\mathrm{SI}} / m_{\mathrm{DM}} \sim \alpha_{D}^{2} / m_{\mathrm{DM}}^{3}$, with $\alpha_{D}=g_{D}^{2} / 4 \pi$. Taking $\alpha_{D} \lesssim \mathcal{O}(1)$, this implies that the DM mass must lie roughly below $500 \mathrm{MeV}$. This bound is much lower if the self-interaction mediator is much heavier than the DM particle.

\section{- Kinematically allowed DM annihilation channels.}

For such a low mass range, DM must necessarily annihilate into one of the few kinematically allowed SM channels: DM DM $\rightarrow \nu \bar{\nu}, \nu \nu, e^{+} e^{-}, \mu^{+} \mu^{-}, \gamma \gamma$ and DMDM $\rightarrow$ $u \bar{u}, d \bar{d}$ (i.e. DM DM $\rightarrow \pi^{+} \pi^{-}, \pi^{0} \pi^{0}$ at the scale under consideration).

\section{- DM must be a gauge singlet.}

This is a consequence of the first requirement. For instance, particles with $\mathrm{SU}(2)_{L} \times$ $\mathrm{U}(1)_{Y}$ quantum numbers and a mass well below the electroweak scale would have been seen in the decay of the $Z$ boson at LEP [28]. There are exceptions to this rule, but they entail some degree of fine tuning, so we will not consider them. An example would have been DM as the CP-even neutral component of a scalar doublet. It can be light and still escape the bound coming from the width of the $Z$ boson if the CP-odd component in the doublet has a mass above $m_{Z}$.

\section{- Extra particles mediating the annihilation are singlets.}

For the same reason, any additional non-singlet particle mediating DM annihilations into SM particles would have to be much heavier than the DM particle, typically above $m_{Z} / 2$ or higher. This would suppress the annihilation cross section by powers of this high mass. As a result in this case we find that the thermal freeze-out could only be obtained for couplings on the verge of non-perturbativity.

In order to illustrate this, let us consider the tree-level annihilation of a Dirac DM particle into neutrinos via the exchange of the neutral component of a $\mathrm{SU}(2)_{L}$ doublet in the t-channel. The annihilation is suppressed by four powers of the mass of the exchanged particle. Concretely, one obtains an annihilation cross section into neutrinos of a given flavor equal to

$$
\left\langle\sigma_{\text {anni }} v\right\rangle=\frac{y^{4}}{32 \pi} \frac{m_{\mathrm{DM}}^{2}}{m_{\phi}^{4}},
$$

where $m_{\phi}$ is the mass of the neutral scalar in the t-channel and $y$ is the Yukawa coupling in the interaction $\mathcal{L}=y \overline{L_{L}} \phi \psi_{\mathrm{DM}}$. For sub-GeV DM and $m_{\phi}$ of order $m_{Z}$, this gives the thermal freeze-out cross section only for quite large Yukawa couplings, at the 
verge of non-perturbativity, ${ }^{2}$ namely, $y \gtrsim 5.6 \cdot\left(100 \mathrm{MeV} / m_{\mathrm{DM}}\right)^{1 / 2} \cdot\left(m_{\phi} / 100 \mathrm{GeV}\right)$. Or in other words, imposing $y \lesssim \sqrt{4 \pi}$ leads to $m_{\mathrm{DM}} \gtrsim 200 \mathrm{MeV} \cdot\left(m_{\phi} / 100 \mathrm{GeV}\right)^{2}$. Also, notice that the same type of Yukawa interactions potentially leads to DM annihilations into charged leptons, and that a thermal rate for that channel is forbidden by indirect detection constraints, as discussed below. As a result of all these, we will not consider any further such kinds of contrived scenarios.

Note that SIDM annihilations into photons are suppressed not only by the loop factor but also, in a similar way, by the large mass of the charged mediator in the loop. The same remarks apply to other processes leading to sharp spectral features, such as virtual internal bremsstrahlung (since they require a charged mediator in the t-channel).

The previous four criteria greatly simplify the discussion and highly limit the number of scenarios where SIDM could freeze out from annihilations into SM particles, as we will see in the following.

\subsection{Four portals to the SM}

In a renormalizable theory, if both the DM and the particle mediating the annihilation process are singlets, they can only communicate with the SM particles via the so-called portals. They correspond to the four possible ways of building, out of SM fields, a gauge singlet operator of dimension less than four [31, 32], namely

$\begin{aligned} \text { Vector portal: } & \overline{\psi_{\mathrm{SM}}} \gamma^{\mu} \psi_{\mathrm{SM}} \\ \text { Kinetic portal: } & F_{Y}^{\mu \nu} \\ \text { Higgs portal: } & H^{\dagger} H \\ \text { Neutrino portal: } & \bar{L} H\end{aligned}$

where $\psi_{\mathrm{SM}}$ is any SM fermionic chiral multiplet, $F_{Y}^{\mu \nu}$ is the hypercharge field strength, $H$ is the SM scalar doublet and $L$ is one of the lepton doublets.

On the one hand, the fermion bilinear can only be coupled in a renormalizable way to a vector boson field, $Z_{\mu}^{\prime}$. On the other hand, the hypercharge field strength can only couple to the field strength of a vector boson, $Z_{\mu \nu}^{\prime}$, through a kinetic mixing interaction

$$
\mathcal{L}=-\frac{\kappa}{2} F_{Y}^{\mu \nu} Z_{\mu \nu}^{\prime}
$$

Thus, from the exchange of a $Z^{\prime}$, both sectors can communicate through either of these two portals or through both.

As for the $H^{\dagger} H$ bilinear, it can couple to any single scalar operator with dimension two. The most general form is

$$
\mathcal{L}=H^{\dagger} H \cdot\left(\mu_{i} \phi_{i}+\lambda_{i j} \phi_{i} \phi_{j}\right)
$$

\footnotetext{
${ }^{2}$ Note that for Majorana or scalar DM, the exchange of a doublet in the t-channel also induces annihilations into neutrinos. However, in those cases, the cross section is even more suppressed than for Dirac DM, because it is proportional to the neutrino masses (see e.g. [29, 30]). In fact, we did not find any viable scenario where DM annihilations into SM fermions are suppressed by only two powers of the mass of the exchanged particle.
} 
where $\phi_{i}$ are singlet scalar fields. Finally, the bilinears $\bar{L} H$ must couple to fermion singlets, i.e. to right-handed neutrinos

$$
\mathcal{L}=y_{\alpha} \overline{L_{\alpha}} H \nu_{R}+\text { h.c. },
$$

All these portals induce annihilations at tree level. In principle, such annihilations can proceed in three ways: from a s- or a t-channel exchange and from a quartic bosonic interaction. We now discuss each case separately:

1. Tree-level annihilation via a $Z^{\prime}$ exchange. Since the $Z^{\prime}$ couples to a pair of SM particles, the annihilation necessarily takes place through a s-channel exchange, either from the vector portal, or from the kinetic portal, or from both. Furthermore, DM naturally self-interacts at an unsuppressed rate via the exchange of the $Z^{\prime}$ boson. Hence, this scenario is particularly attractive and minimal. We will discuss it in detail in section 3. It differs from previous SIDM studies involving $Z^{\prime}$ bosons by the fact that here DM is lighter than such particles, and thus does not annihilate into a pair of them but into SM particles through the s-channel exchange of a $Z^{\prime}$. Notice that models with $\mathrm{MeV}$ DM coupled to a heavier $Z^{\prime}$ boson have also been considered in contexts different from SIDM (See e.g. [33, 34] for its implications on the galactic $511 \mathrm{keV}$ line).

2. Tree-level annihilation via the Higgs portal. If a field $\phi$ entering in the Higgs portal above has no linear interactions -in particular no vacuum expectation value $\langle\phi\rangle$ and no term $\mu \phi H^{\dagger} H$ in the Lagrangian- it can only communicate to the SM through the interaction $\mathcal{L}=\lambda \phi \phi H^{\dagger} H$.

In this case, this field could be a DM candidate and annihilate through a Higgs boson exchange into light SM particles. However, taking into consideration that (i) the Higgs boson can not decay into DM with a large rate (in order to avoid the LHC bound on its invisible decay width), (ii) the Yukawa couplings of light fermions are very small, and (iii) the Higgs boson is much heavier than the sub-GeV DM candidate discussed here, we conclude the exchange of a Higgs boson can not mediate annihilations processes fast enough in order to lead to the observed relic density.

Instead, if there is a Higgs portal interaction linear in $\phi$ (i.e. $\mu \neq 0$, or $\lambda \neq 0$ when $\langle\phi\rangle \neq 0$ ), after electroweak symmetry breaking, the scalar field $\phi$ mixes with the SM model scalar and inherits its Yukawa couplings to ordinary fermions. It is thus unstable and we need an additional particle as DM candidate, which annihilates into SM fermions via the scalar portal. The tree-level annihilations of such candidate can take place via the exchange of the two scalar mass eigenstates in the s-channel: the Higgs boson and the other scalar arising from the mixing. The former case is excluded in the (i)-(iii) way above. The latter case is also excluded because, even if the other scalar arising from the mixing is lighter, the corresponding annihilations turn out to be still quite suppressed because its interactions are still proportional to the small Higgs Yukawa couplings. Thus, unless we sit on the $m_{\phi} \simeq 2 m_{\mathrm{DM}}$ resonance to enhance the annihilation process, the relic density cannot be accounted via the freeze-out mechanism and this scenario is therefore not viable. 
3. Tree-level exchange via the neutrino portal. The neutrino portal requires one or more right-handed neutrinos. Since it necessarily induces a mixing of these particles with the SM neutrinos, this portal offers the possibility of having DM annihilations into active neutrinos. At tree level, for scalar DM as well as fermion DM, there are three ways to induce such an annihilation, two in the s-channel (via the exchange of a scalar or a vector particle) and one in the t-channel. In all these cases, this requires the existence of an extra particle in addition to the DM and singlet neutrinos. However, the resulting neutrino mixing is highly bounded from above by neutrinomass constraints, and the corresponding annihilation cross section turns out to be too small. Thus, the neutrino portal does not work for our purposes either.

\subsection{Surpassing indirect detection constraints}

DM annihilations into SM particles can potentially produce a significant flux of cosmic rays, specially if they are produced in astrophysical systems where the DM concentration is known to be very high (see e.g. ref. [35]). Likewise, such annihilations also lead to distortions of the CMB spectrum [36-38] or to a departure from the predictions of standard Big Bang Nucleosynthesis (BBN) [39, 40]. The non-observation of these phenomena leads to stringent bounds on annihilations cross sections, specially for sub-GeV DM. In fact, one finds that an annihilation cross section into SM particles around the thermal freeze-out value is excluded for such masses, except in two cases:

- If DM annihilates almost exclusively into neutrinos [41]. The neutrino portal would have been interesting in this respect because it gives rise to such situation naturally. Nonetheless, it does not work in the context of SIDM, as mentioned above.

- If DM annihilations are velocity-suppressed. In this case all fluxes are suppressed because in all the systems from which the bounds are derived, DM moves with very small velocities compared to the freeze-out epoch [42-46]. More quantitatively, for velocitysuppressed DM annihilations, the cross section can be expanded as $\sigma_{\text {anni }} v=b v^{2}$, where $v$ is the relative DM velocity at a given epoch. The observed DM density $\Omega h^{2}$ fixes the quantity $b$. Using the instantaneous freeze-out approximation as reported in ref. [47], one gets the following relic abundance

$$
\Omega h^{2}=\left(\frac{1.07 \times 10^{9} \mathrm{GeV}^{-2}}{3 g_{*}\left(x_{f}\right)^{1 / 2} M_{\mathrm{Pl}} b}\right) n x_{f}^{2},
$$

where $x_{f} \approx 20$ is the usual inverse freeze-out temperature, $n=1$ for self-conjugate DM and $n=2$ in the opposite case. Taking the relic density equal to $\Omega h^{2}=$ $0.1199 \pm 0.0027$ [48], the previous procedure leads to values of $b$ of about $10^{-25} \mathrm{~cm}^{3} / \mathrm{s}$ for sub-GeV DM. For such values, scenarios with velocity-suppressed DM annihilations are not constrained by indirect searches. Both photo-dissociation of ${ }^{4} \mathrm{He}$ and photon-decoupling processes happen when the DM particles are already highly nonrelativistic. Therefore, $\mathrm{CMB}$ and $\mathrm{BBN}$ bounds are irrelevant here and the most stringent constraints can only come from DM indirect searches in dark halos at very low redshifts. However, even there, current experiments give upper bounds on $b v^{2}$ of 
around $10^{-28}-10^{-27} \mathrm{~cm}^{3} / \mathrm{s}$ [49] (with $v \lesssim 10^{-2}$ in dark halos, as given by cosmological simulations), leaving velocity-suppressed annihilations cross sections unconstrained.

In practice, a velocity suppression in the annihilation process means that the s-wave piece of the corresponding cross section is not allowed. For the portals which have been found to be viable above, i.e. the vector and kinetic portals, this is only possible in specific cases. To see that, suppose that DM annihilation takes place via the s-wave, i.e. with orbital angular momentum $L=0$. In order to exchange a $Z^{\prime}$, we must have a state with total angular momentum $J=1$, or equivalently total spin $S=1$. This is not possible for scalar or Majorana DM since they both lead to $S=0$; the state $S=1$ is symmetric for a pair of fermions in the $L=0$ configuration and is thus banned for Majorana particles. As a result, if we couple the $Z^{\prime}$ boson to scalar or Majorana DM particle, we naturally obtain velocity-suppressed annihilations and evade indirect detection bounds.

Note that both of these scenarios can hardly be probed by high-energy colliders. For example, missing-energy searches at LHC are able to exclude thermal freeze-out mechanism for $m_{\mathrm{DM}} \lesssim O(10) \mathrm{GeV}$ if $m_{Z^{\prime}} \gtrsim 50 \mathrm{GeV}$ [50]. But if the mediator is also light, thermal freeze-out of DM only requires much weaker couplings with SM particles, which is well beyond the reach of high-energy collider experiments. This has been shown in various so-called simplified model studies (for a recent analysis, see [51]). In contrast, it is well known that data-intensive experiments at relatively low energy, as well as other precision measurements, may provide very strong bounds for $Z^{\prime}$-portal models at the scale below $\mathrm{GeV}$ [32]. This has been discussed in the previous literature $[52,53]$ and will be investigated in the SIDM framework for both Majorana and scalar DM in the next section.

In conclusion, the previous natural list of constraints and criteria point towards a unique scenario with two variants: Majorana or scalar DM annihilating into light SM leptons or quarks through a heavier spin-1 particle exchange.

\section{Scenarios with a $Z^{\prime}$ boson}

As said above, a $Z^{\prime}$ can be exchanged between the DM particle and the SM sector from the vector portal, the kinetic mixing portal, or both. In either case, we assume the $Z^{\prime}$ to be associated to a $\mathrm{U}(1)_{D}$ gauge interaction with a mass originating from the Brout-EnglertHiggs or the Stueckelberg mechanisms.

If some of the light SM particles are charged under the $\mathrm{U}(1)_{D}$ group and if in addition there is no kinetic mixing, DM communicates with the SM sector only through the vector portal. Provided the $\mathrm{U}(1)_{D}$ gauge coupling and the corresponding charges are of order one (as it is the case for the known gauge groups), this possibility is highly constrained by collider experiments. In particular, the bound $m_{Z^{\prime}}>2.1 \mathrm{TeV}$ holds if the $Z^{\prime}$ sizably couples to SM leptons [54]. For a leptophobic $Z^{\prime}$, the bound is weaker, but in general still requires $m_{Z^{\prime}}$ heavier than few hundred GeVs, depending on its exact couplings to quarks [55-57]. Such heavy $Z^{\prime}$ can not induce DM annihilations with perturbative couplings, thus we will not consider this portal any further (although it could certainly work in special cases where the relevant gauge couplings have sufficiently small values). 
In the following, we will consider the opposite option, where all SM particles have no $\mathrm{U}(1)_{D}$ charges, but where there is a non-zero kinetic mixing interaction, as given in eq. (2.2), so that the communication of both sectors solely occurs through this portal.

This leads to a highly predictive and minimal scenario, in which all $Z^{\prime}$ couplings to SM particles are known up to the overall multiplicative kinetic mixing parameter. Concretely, after electroweak symmetry breaking, eq. (2.2) gives rise to the following $Z^{\prime}$ interactions

$$
\mathcal{L} \supset g_{D} J_{\mathrm{DM}}^{\mu} Z_{\mu}^{\prime}+\epsilon e \bar{J}_{\mathrm{EM}}^{\mu} Z_{\mu}^{\prime}+\epsilon^{\prime} g_{Z} \bar{J}_{\mathrm{Z}}^{\mu} Z_{\mu}^{\prime},
$$

where $J_{\mathrm{DM}}^{\mu}, J_{\mathrm{EM}}^{\mu}$ and $J_{\mathrm{Z}}^{\mu}$ are the dark, the QED and the weak neutral currents. The exact expression of $J_{\mathrm{DM}}^{\mu}$ depends on the dark matter spin, and will be given separately for each case below. Also, the vector boson couplings to the SM currents are given, to leading order in the kinetic mixing parameter, by [58]

$$
\epsilon \approx \cos \theta_{\mathrm{W}} \kappa, \quad \epsilon^{\prime} \approx \frac{m_{Z^{\prime}}^{2}}{-m_{Z}^{2}+m_{Z^{\prime}}^{2}} \sin \theta_{\mathrm{W}} \kappa,
$$

where $\theta_{\mathrm{W}}$ is the Weinberg angle. It follows that $\epsilon \gg \epsilon^{\prime}$ for $m_{Z} \gg m_{Z^{\prime}}$. We can thus safely neglect the interaction term involving $J_{Z}^{\mu}$. This in turn means that annihilations into neutrinos are negligible and only final states including light charged leptons or quarks can be responsible for the DM freeze-out. Furthermore, this implies that the DM particle must be heavier than the electron.

\subsection{Majorana dark matter}

If $\mathrm{DM}$ is made of a Majorana fermion $\chi$, their current coupling it to the $Z^{\prime}$ is given by $J_{\mathrm{DM}}^{\mu}=\bar{\chi} \gamma^{\mu} \gamma^{5} \chi$. For the DM mass range of interest, the annihilation channels are $\chi \chi \rightarrow Z^{* *} \rightarrow \bar{f} f$, with $f$ an electron, a muon, an up quark or a down quark (i.e. pions for the last two cases). For a given fermion of electric charge $q_{f}$ and color $N_{f}$, the annihilations cross section is given by

$$
\left\langle\sigma_{\mathrm{anni}} v\right\rangle \simeq \frac{16 \pi \epsilon^{2} \alpha \alpha_{D} \sum_{f} N_{f} q_{f}^{2}\left(1-r_{f}\right)^{1 / 2}\left(2+r_{f}\right) v^{2}}{3 m_{\chi}^{2}\left(\left(r_{Z^{\prime}}-4\right)^{2}+r_{Z^{\prime}}^{2} \Gamma_{Z^{\prime}}^{2} / m_{Z^{\prime}}^{2}\right)},
$$

with $r_{a}=m_{a}^{2} / m_{\chi}^{2}$. Summing over all kinematically allowed channel, the relic density is given by eq. (2.5). We work under the approximation that, for $m_{\chi}>m_{\pi}$, terms in eq. (3.3) associated to the up and down quarks give the inclusive cross section into pions.

As for the self-interaction hypothesis, we have

$$
\sigma_{\mathrm{SI}} / m_{\chi} \simeq 512 \pi \alpha_{D}^{2} m_{\chi} / m_{Z^{\prime}}^{4} \simeq 1 \mathrm{~cm}^{2} / \mathrm{g},
$$

where the low velocity limit has been taken. Note that the non-observation of an offset between the mass distribution of DM and galaxies in the Bullet Cluster has been claimed to constrain the self-interacting cross section, $\sigma_{\mathrm{SI}} / m_{\mathrm{DM}}<1.25 \mathrm{~cm}^{2} / \mathrm{g}$ at $68 \%$ CL [59-61]. However, recent simulations suggest that stronger self-interactions are still allowed [13, 62]. In the following, we will always take $1 \mathrm{~cm}^{2} / \mathrm{g}$ as a benchmark value for $\sigma_{\mathrm{SI}} / m_{\mathrm{DM}}$. Modifying it by a factor of a few would only affect our conclusions mildly. 

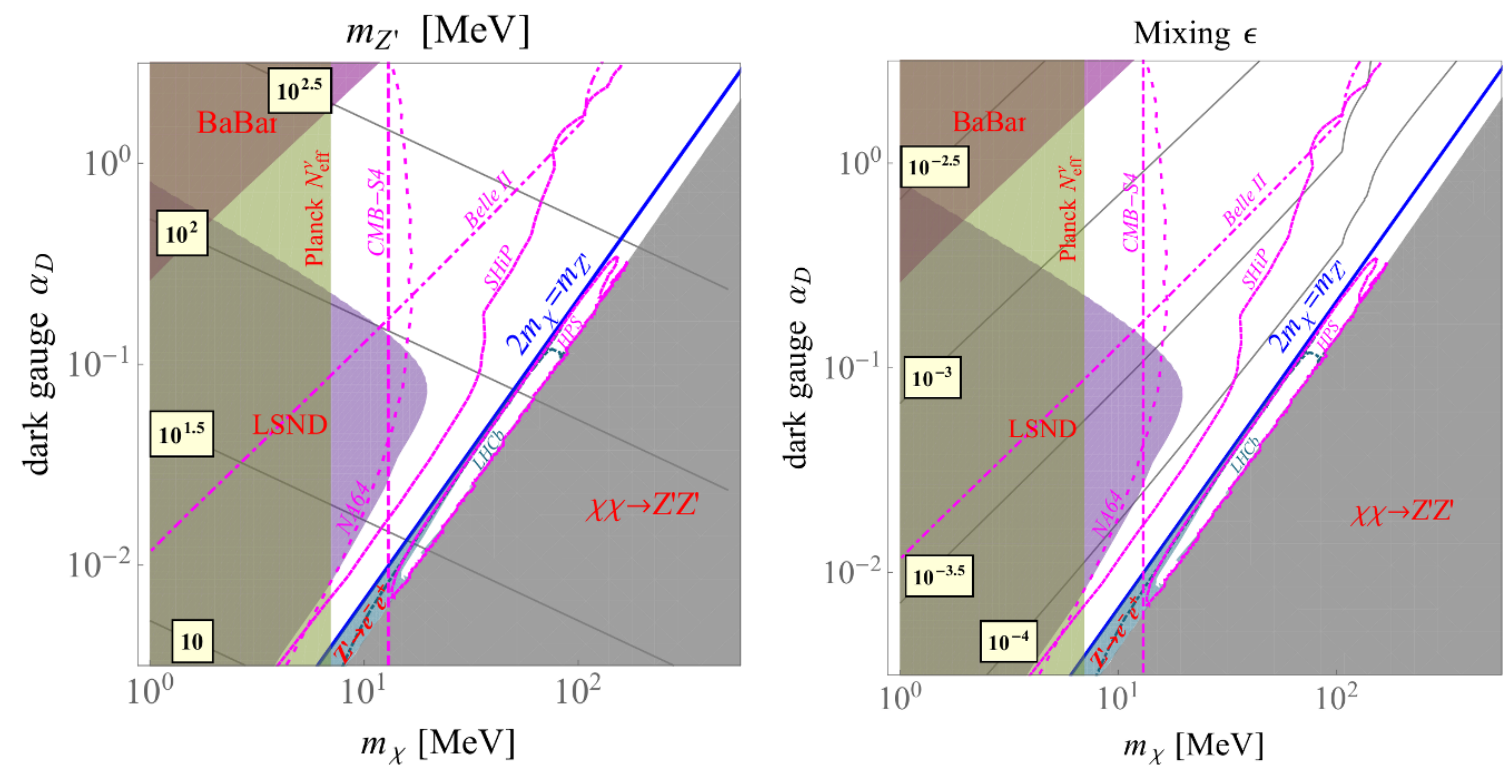

Figure 1. $Z^{\prime}$ portal for Majorana DM. As a function of the DM mass $m_{\chi}$ and dark coupling $\alpha_{D}$, the solid contour lines show the values of $Z^{\prime}$ mass (left) and kinetic mixing parameter $\epsilon$ (right) satisfying the relic density and the self-interaction constraints, as given in eqs. (2.5) and (3.4). All shaded regions are experimentally excluded in various ways (see text for details). In the shaded region at the right-bottom corner, the dark annihilation $\chi \chi \rightarrow Z^{\prime} Z^{\prime}$ is too fast to account for the DM abundance. Non-solid (colored) lines show the expected sensitivities of future experiments.

The relic density and self-interactions constraints just mentioned fix $m_{Z^{\prime}}$ and $\epsilon$ as functions of DM mass $m_{\chi}$ and dark fine structure constant $\alpha_{D}$. Figure 1 shows the values we obtain by following this procedure.

As said above, to prevent a fast DMDM $\rightarrow Z^{\prime} Z^{\prime}$ annihilation leading to a too suppressed relic density, one assumes $m_{Z^{\prime}} \gtrsim m_{\mathrm{DM}}$. More exactly, this requirement rather leads to $m_{Z^{\prime}} \gtrsim 1.6 m_{\mathrm{DM}}$ after taking into account eq. (3.4). All values not satisfying this requirement are shaded in grey in figure 1 . For a precise value of $m_{Z^{\prime}} / m_{\mathrm{DM}}$ around 1.6, the annihilation rate is Boltzmann suppressed $\left(\propto e^{-2 m_{Z^{\prime}} / T}\right)$ just enough to lead to the observed relic density. In this case, both self-interactions and annihilations constraints are accounted for by the hidden sector interaction and the condition on the connector $\epsilon$ is that it has to be small enough to play only a subleading role in the annihilation process. This way of accounting for both constraints in the hidden sector by means of a threshold effect [47] has been proposed in ref. [63] and is operative here. For smaller (larger) values of $m_{Z^{\prime}} / m_{\mathrm{DM}}$, the DMDM $\rightarrow Z^{\prime} Z^{\prime}$ annihilation rate is very quickly far too fast (slow) to account for the relic density. In the later case, $m_{Z^{\prime}} / m_{\mathrm{DM}} \gtrsim 1.6$, the connector interaction can nevertheless account for it. This is the scenario we consider, which leads to a whole allowed region in figure 1.

Not surprisingly, figure 1 reflects the disparity of both cross sections. On the one hand, the self-interaction constraint requires a cross section, eq. (3.4), which is not suppressed in any way, i.e. a relatively large value of $\alpha_{D}$ and a relatively light mediator, $m_{Z^{\prime}} \lesssim \mathcal{O}(1) \mathrm{GeV}$. On the other hand, for sub-GeV DM the relic density constraint, eq. (3.3), requires a 
suppression of the annihilation cross section. This can only arise from a suppressed portal, i.e. mixing parameter $\epsilon$. This way of decoupling both cross sections is an easy way to account for the big difference between them: the annihilation rate is naturally suppressed with respect to the self-interactions because the portal, which enters only in the annihilation cross section, is very small. Note that, as figure 1 shows, for $m_{Z^{\prime}} \sim 2 m_{\chi}$ the annihilation cross section displays a resonance, requiring even smaller values of $\epsilon$.

There is a long list of constraints applying to this scenario. The most relevant ones are shown on figure 1. These are:

- Invisible decay of $Z^{\prime}$ in low energy experiments. Due to the large value of $\alpha_{D}$ and small value of $\epsilon$, when $m_{Z^{\prime}} \gtrsim 2 m_{\chi}$ (above the blue lines in figure 1 ), the $Z^{\prime}$ decays invisibly with a branching ratio close to one. Hence, at colliders, the $Z^{\prime}$ cannot be seen directly and the best way to detect it is from the observation of initial state radiation and missing energy. Note that the cross section for such a signal depends only on $m_{Z^{\prime}}$ and on the size of the couplings between the $Z^{\prime}$ and the SM particles in the initial state, i.e. on the size of the $\epsilon$ parameter. The BaBar collaboration -searching for the decay of $\Upsilon(3 S)$ to mono-photon and invisible particles- has constrained the coupling between $Z^{\prime}$ and SM particles for $m_{Z^{\prime}} \lesssim 7.2 \mathrm{GeV}$ [64, 65]. This constraint is shown as a shaded region in figure 1. In addition, the Belle II experiment, which should start taking data after 2018 [66], has the potential to improve the constraint on $\epsilon$ by about one order of magnitude. In figure 1, the dot-dashed line in the left-top corner shows the corresponding projected sensitivity, adapted from the mono-photon search done in ref. [65].

Likewise, by studying the process $e Z \rightarrow e Z Z^{\prime}$, the fixed-target experiment NA64 at CERN SPS will be able to probe dark photon decays into invisible particles [67]. The corresponding projected sensitivity (associated to $10^{11}$ incident electrons) is shown in figure 1.

Interestingly, events associated to the invisible decay of a $Z^{\prime}$ boson may be recorded in neutrino experiments as well. Concretely, if $m_{Z^{\prime}}<m_{\pi^{ \pm}}$, depending on its couplings to SM fermions, the $Z^{\prime}$ boson might be produced in pion decays and quickly disintegrate into invisible particles, i.e. DM in our scenario. In turn, the DM particles might collide against the electron target, leading to detectable scattering events, similar to the ones induced by neutrinos. Thus, the observed number of such events can be used to constrain our scenario if the $Z^{\prime}$ is lighter than the pion(s). Using this, the LSND data provides the strongest constraint for very light dark matter [68]. This is shown by the shaded region labeled as "LSND" in figure 1. Moreover, using the same search strategy, the SHiP experiment will improve this constraint as shown in figure 1 . The projected sensitivity in the plot corresponds to a yield of 10 electron-scattering events [69].

Also, it has been pointed out that the experiment E137 performed at SLAC three decades ago provides similar constraints on dark photons [70]. In most of the parameter region of our interest, they are less stringent than those of LSND and are thus not shown in our figure. 
- Precision test bounds. When $m_{Z^{\prime}} \gtrsim 2 m_{\chi}$, this scenario is also constrained by missing $E_{T}$ searches at higher energy accelerators, such as LEP and LHC. The current bound is $\epsilon \lesssim 0.23$ for $m_{Z^{\prime}}$ below few $\mathrm{GeV}$ [65]. As $Z^{\prime}$ mixes with the SM $Z$ boson, $Z$ pole precision measurements also constrain the mixing parameter, giving $\epsilon \lesssim 0.3$ [71]. Moreover, anomalous magnetic moment measurements of the electron and the muon, as well as neutron-nucleus scattering measurements [72], also lead to upper bounds on $\epsilon$. Nevertheless, all these precision bounds are looser than other constraints and we do not show them on figure 1 .

- Visible decay of $Z^{\prime}$. For the case $m_{Z^{\prime}} \lesssim 2 m_{\chi}$ (below the blue lines in figure 1 ), the $Z^{\prime}$ boson decays into pairs of SM fermions. This possibility is extensively considered in experiments looking for dark photons. For instance, searching for the process $\pi^{0} \rightarrow \gamma Z^{\prime}$ followed by $Z^{\prime} \rightarrow e^{+} e^{-}$, the NA48/2 collaboration has excluded $\epsilon \gtrsim 8 \times 10^{-7}$ at $90 \%$ CL when the $Z^{\prime}$ boson is lighter than the neutral pion [73, 74]. We refer to ref. [32] for a recent review on this constraint and others from beam-dump/collider experiments. This leads to the exclusion of the shaded region labeled as " $Z$ ' $\rightarrow e^{-} e^{+}$" in figure 1. One can see that there still exists a large unconstrained region between $\mathcal{O}\left(10^{-5}\right) \lesssim \epsilon \lesssim \mathcal{O}\left(10^{-2}\right)$ for DM masses of few tens of MeVs. Independently of selfinteraction constraints, this feature is also shown in figure 6 of the review [32].

Figure 1 also shows the sensitivities expected to be reached in the future by various experiments: from the proposed Heavy Photon Search (HPS) [75], looking for leptonic decays of a dark $Z^{\prime}$ boson, and from the dark photon search at the run 3 of LHCb using charm meson decays [76]. Clearly, these experiments offer real prospects to probe our scenario.

- Cosmological bounds. As DM annihilation is velocity suppressed, it does not directly change BBN predictions or the CMB spectrum, as explained above. It can nevertheless have an indirect effect from the fact that -after neutrino decoupling at about $1.5 \mathrm{MeV}$ [77]- late annihilations of DM into electron-positron pairs, may reheat the thermal bath of photons with respect to the cosmic neutrino bath. This leads to a relatively colder neutrino sector at the recombination time. Taking $N_{\text {eff }}^{\nu} \gtrsim 2.9$ from Planck, we obtain that $m_{\chi} \gtrsim 7 \mathrm{MeV}$, as shown by the left shaded region "Planck" in figure 1 [78]. Note that assuming an earlier neutrino decoupling would lead to a stronger bound. Proposed CMB precision experiments, referred to as "CMB-S4", intend to reduce the uncertainty on $N_{\text {eff }}^{\nu}$ to $0.01[79,80]$. This would lead to a stronger lower bound on $m_{\chi}$ of about $12 \mathrm{MeV}$.

Note that observations of supernova explosions only constrain small values of $\epsilon$ that are irrelevant here. In fact, kinetic mixing values larger than $\mathcal{O}\left(10^{-6}\right)$ are enough to avoid that most of $Z^{\prime}$ and DM particles escape the supernova core. Thus, the predictions of our scenario regarding supernovae are indistinguishable from those of the SM [81].

- Direct searches. The scattering of Majorana DM particles off nucleons is velocitysuppressed if such process is induced by the exchange of a $Z^{\prime}$ boson coupled to a 
vector current of SM fermions. That is the case of the present scenario because, as said above, for $m_{Z^{\prime}} \ll m_{Z}$, the neutral current $J_{Z}^{\mu}$ is approximately decoupled from the portal interactions. Thus, this scenario can be hardly constrained by current direct detection experiments.

- Indirect searches. As discussed at length previously, the p-wave annihilation channels responsible for the relic density are suppressed by at least two powers of the DM velocity. Moreover, other processes such as virtual internal bremsstrahlung or one-loop annihilations into photons are suppressed by the mass of the charged mediators that could induce them. One might think that for $m_{\chi} \geq m_{\pi} / 2$, the processes $\chi \chi \rightarrow \pi^{0} \gamma$ are relevant. However, they do not arise in the s-wave configuration as they require angular momentum $J=1$. Consequently, as already anticipated in section 2, this scenario can not be probed by indirect searches of DM.

From this list of constraints, we conclude that Majorana DM coupled to a slightly heavier $Z^{\prime}$ boson provides a viable model of self-interacting DM, that is still allowed within a relatively large region of the parameter space. As shown in figure 1, the preferred DM masses lie around a few tens of MeV. While the HPS experiment and LHCb will probe a large fraction of the parameter space where the $Z^{\prime}$ boson decays visibly, Belle-II and the SHiP experiment at the CERN SPS will probe part of the region $2 m_{\chi} \lesssim m_{Z^{\prime}}$, where it decays invisibly.

Before closing this section, we would like to comment on simple UV completions of this scenario. Since Majorana fermions can not carry any charge, their $Z^{\prime}$ axial current can only arise from the spontaneous symmetry breaking of the $\mathrm{U}(1)_{D}$ symmetry. For instance, the vev of a single scalar $S$ with twice the $\mathrm{U}(1)_{D}$ charge of the chiral DM fermion can induce both the Majorana mass of this fermion and the mass of the $Z^{\prime}$. Also, anomaly cancellation requires extra fermions charged under $\mathrm{U}(1)_{D}$, introduced either in a chiral way (with several extra Weyl fermions [83]) or in a vector-like way (assuming a chiral partner for the DM field, at the price of allowing a new mass scale). The DM can be lighter than all other hidden sector particles if its Yukawa coupling to $S$ is relatively small with respect to the couplings determining the masses of $S, Z^{\prime}$ and the extra fermions. This is always possible in the parameter space of our interest, as shown in figure 1 . In this case, these additional particles do not change the phenomenology of interest in this work. The extra fermions decay into DM particles and their participation in the freeze-out is suppressed. Similarly, the scalar does not affect the freeze-out, and its contribution to DM self-interactions is suppressed by powers of the Yukawa coupling. Finally, the scalar $S$ must not strongly mix with the SM Higgs boson to satisfy bounds from the Higgs invisible decay and other DM searches.

\subsection{Scalar dark matter}

If DM is made of a scalar $S$ annihilating into SM particles via a s-channel exchange of $Z^{\prime}$ bosons, one obtains a scenario similar to the Majorana case, except for three important 
differences: DM is not its own antiparticle, ${ }^{3}$ there is an extra source of self-interaction for the DM and the direct detection rate is not anymore velocity suppressed.

First of all, let us look at the annihilation process, which is induced by the current $J_{\mathrm{DM}}^{\mu}=i\left(S^{*} \partial_{\mu} S-S \partial_{\mu} S^{*}\right)$. In the non-relativistic limit, the corresponding cross section is given by

$$
\left\langle\sigma_{\text {anni }} v\right\rangle \simeq \frac{4 \pi \epsilon^{2} \alpha \alpha_{D} \sum_{f} N_{f} q_{f}^{2}\left(1-r_{f}\right)^{1 / 2}\left(2+r_{f}\right) v^{2}}{3 m_{S}^{2}\left(\left(r_{Z^{\prime}}-4\right)^{2}+r_{Z^{\prime}}^{2} \Gamma_{Z^{\prime}}^{2} / m_{Z^{\prime}}^{2}\right)},
$$

Summing over all kinematically allowed channels in the same way as for the Majorana case above, eq. (2.5) fixes the DM relic density.

As for the DM self-interactions, they are induced by the exchange of the $Z^{\prime}$ boson, and possibly by an additional $\mathcal{L}_{\mathcal{S}} \supset-\lambda_{S}\left(S^{*} S\right)^{2}$ quartic coupling contribution. Due to the fact that DM is not is own antiparticle, there are several self-interaction channels, namely, $S S \leftrightarrow S S, S S^{*} \leftrightarrow S S^{*}$ and $S^{*} S^{*} \leftrightarrow S^{*} S^{*}$. The corresponding averaged cross section in the non-relativistic limit is

$$
\frac{\sigma_{\mathrm{SI}}}{m_{S}}=\frac{3 \lambda_{S}^{2}}{16 \pi m_{S}^{3}}+\frac{6 \pi \alpha_{D}^{2} m_{S}}{m_{Z^{\prime}}^{4}} .
$$

For the case where one assumes a negligible value of the quartic coupling, the left panel of figure 2 shows, as a function of $m_{S}$ and $\alpha_{D}$, the values of $m_{Z^{\prime}}$ that one needs in order to fulfill both the relic density constraint and the benchmark $\sigma_{\mathrm{SI}} / m_{\mathrm{DM}}=1 \mathrm{~cm}^{2} / \mathrm{g}$. Likewise, the right panel of figure 2 shows the corresponding situation when we switch on the scalar coupling, taking $\lambda_{S}=0.1$ as a sample value.

In the latter case, as shown in the right panel of figure 2, the self-interaction hypothesis precludes too light dark matter candidates independently of the value of $\alpha_{D}$, because the scalar coupling contribution to the self-interaction cross section scales as $1 / m_{S}^{2}$. Also, note that having a large value of $\lambda_{S}$ at such a low scale may give rise to a Landau pole below the electroweak scale (unless there are extra low energy degrees of freedom in the hidden sector contributing negatively to the $\beta$ function of this coupling ${ }^{4}$ ). For example in a pure $\lambda_{S} S^{4}$ theory, a value $\lambda_{S}=0.1$ at $m_{S} \sim 10-100 \mathrm{MeV}$ scale leads to a Landau pole around the electroweak scale.

As mentioned at the beginning of this section, unlike for Majorana particles, the scalar case does not lead to velocity-suppressed direct detection cross sections. Although currently sub-GeV DM is almost unconstrained by direct detection experiments, such an unsuppressed rate may lead to potential tests in the future. Experiments searching for nuclear recoil are not so promising in this regard. For example, the most optimistic case for our purpose is the future SNOLAB experiment which will be able to probe DM particles with masses down to $0.5 \mathrm{GeV}$. However, a signal could be seen in experiments searching for DM-electron collision. For scalar DM communicating with the SM via a $Z^{\prime}$-portal,

\footnotetext{
${ }^{3}$ For the sake of simplicity, we assume DM to be symmetric, i.e. the abundance of $S$ and $S^{*}$ are taken equal.

${ }^{4}$ Notice that a Landau pole can also develop for the scalar $S$ introduced in the UV completion of the Majorana scenario above. Nevertheless, such Landau pole can be easily avoided because there the heavier fermions introduced for anomaly cancellation provide such negative contribution.
} 

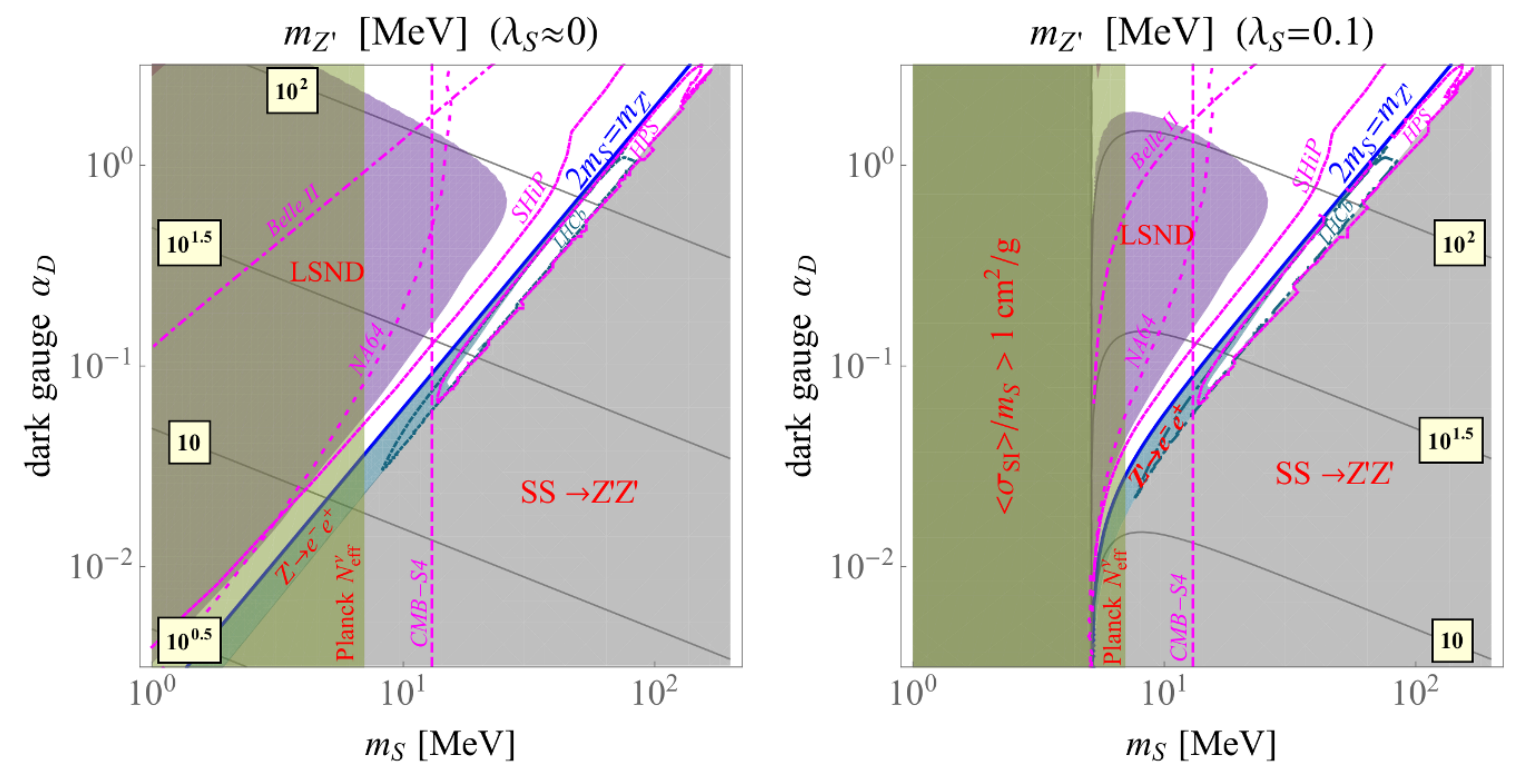

Figure 2. $Z^{\prime}$ portal for scalar DM. As a function of the DM mass $m_{S}$ and dark coupling $\alpha_{D}$, the solid lines show the $Z^{\prime}$ mass satisfying the relic density and the self-interaction constraints, as given in eqs. (2.5) and (3.4), for two choices of the scalar self-coupling $\lambda_{S}$. Results are similar to the Majorana case above, specially for $\lambda_{S} \sim 0$. In the shaded region at right-bottom corner, the dark freeze-out from $S S \rightarrow Z^{\prime} Z^{\prime}$ is too fast to account for the DM abundance. In the right panel, a lower bound $m_{S} \gtrsim 5.4 \mathrm{MeV}$ holds due to the non-zero quartic coupling contribution to the self-interactions. Note that future direct detection experiments with semiconductor targets are expected to probe all the allowed region [82].

this has been studied in for XENON10 [84, 85]. Here, the collision cross section is given by $\sigma_{e} \sim 16 \pi \epsilon^{2} \alpha_{D} \alpha m_{\mathrm{e}}^{2} / m_{Z^{\prime}}^{4}$. Lastly, future experiments with semiconductor targets are expected to be able to probe the whole parameter region allowed today [82].

\section{Conclusions}

In this work, we have shown that it is possible for a self-scattering DM particle (with a strength capable of addressing the small scale structure problems of the CDM paradigm) to freeze out dominantly from annihilations into SM particles.

We have argued that this is only possible if the DM mass lies below the GeV scale. Barring large fine-tuning, this immediately implies that DM must be a singlet of the SM gauge group. The same remark applies for any particle mediating the annihilation process, because otherwise such mediator would need to be around the electroweak scale or above, and the corresponding annihilation rates would be suppressed. These facts together imply that the DM and the SM sectors must be connected through one or several of the four SM singlet portal interactions, associated to a scalar boson, a right-handed neutrino and a $Z^{\prime}$ massive gauge boson.

We have shown that only the option of a $Z^{\prime}$ boson coupled to Majorana or scalar DM passes all the experimental constraints. From its simplicity and the fact that it does not 
require any special tuning, this scenario constitutes an attractive way to accommodate both DM large self-interactions and the relic density constraint. Here, the huge difference between the self-interaction and annihilation cross sections is not due to any special mechanism taking place; it is simply due to the fact that the portal interaction, which enters in the annihilation but not in the self-interaction, is suppressed. Furthermore, this scenario offers possibilities of particle physics tests.

Quantitatively, figure 1 (for the Majorana case) and figure 2 (for the scalar case) summarize the various constraints and future possibilities of testing it or ruling it out. For the scalar case, in addition to the constraints shown in figure 2, semiconductor target direct detection experiments have the potential to probe all the parameter space allowed today.

\section{Acknowledgments}

We thank Julian Heeck and Josh Ruderman for useful discussions. The work of C.G.C. and T.H. is supported by the FNRS, by the IISN and by the Belgian Federal Science Policy through the Interuniversity Attraction Pole P7/37.

Open Access. This article is distributed under the terms of the Creative Commons Attribution License (CC-BY 4.0), which permits any use, distribution and reproduction in any medium, provided the original author(s) and source are credited.

\section{References}

[1] M. Boylan-Kolchin, J.S. Bullock and M. Kaplinghat, Too big to fail? The puzzling darkness of massive Milky Way subhaloes, Mon. Not. Roy. Astron. Soc. 415 (2011) L40 [arXiv: 1103.0007] [INSPIRE].

[2] M. Boylan-Kolchin, J.S. Bullock and M. Kaplinghat, The Milky Way's bright satellites as an apparent failure of $\Lambda$ CDM, Mon. Not. Roy. Astron. Soc. 422 (2012) 1203 [arXiv:1111.2048] [INSPIRE].

[3] M.G. Walker and J. Penarrubia, A Method for Measuring (Slopes of) the Mass Profiles of Dwarf Spheroidal Galaxies, Astrophys. J. 742 (2011) 20 [arXiv:1108.2404] [INSPIRE].

[4] R. Kuzio de Naray and K. Spekkens, Do Baryons Alter the Halos of Low Surface Brightness Galaxies?, Astrophys. J. 741 (2011) L29 [arXiv:1109.1288] [INSPIRE].

[5] D.H. Weinberg, J.S. Bullock, F. Governato, R. Kuzio de Naray and A.H.G. Peter, Cold dark matter: controversies on small scales, in proceedings of the Sackler Colloquium: Dark Matter Universe: On the Threshhold of Discovery, Irvine, U.S.A., October 18-20 2012 [Proc. Nat. Acad. Sci. 112 (2014) 12249] [arXiv:1306. 0913] [InSPIRE].

[6] D.N. Spergel and P.J. Steinhardt, Observational evidence for selfinteracting cold dark matter, Phys. Rev. Lett. 84 (2000) 3760 [astro-ph/9909386] [INSPIRE].

[7] B.D. Wandelt, R. Dave, G.R. Farrar, P.C. McGuire, D.N. Spergel and P.J. Steinhardt, Selfinteracting dark matter, in proceedings of the 4 th International Symposium on Sources and Detection of Dark Matter in the Universe (DM 2000), Marina del Rey, California, U.S.A., February 23-25 2000, D.B. Cline ed., Springer-Verlag, Berlin Germany (2001), pp. 263-274 [astro-ph/0006344] [INSPIRE]. 
[8] M. Vogelsberger, J. Zavala and A. Loeb, Subhaloes in Self-Interacting Galactic Dark Matter Haloes, Mon. Not. Roy. Astron. Soc. 423 (2012) 3740 [arXiv:1201.5892] [INSPIRE].

[9] M. Rocha et al., Cosmological Simulations with Self-Interacting Dark Matter I: Constant Density Cores and Substructure, Mon. Not. Roy. Astron. Soc. 430 (2013) 81 [arXiv: 1208.3025] [INSPIRE].

[10] A.H.G. Peter, M. Rocha, J.S. Bullock and M. Kaplinghat, Cosmological Simulations with Self-Interacting Dark Matter II: Halo Shapes vs. Observations, Mon. Not. Roy. Astron. Soc. 430 (2013) 105 [arXiv:1208.3026] [INSPIRE].

[11] J. Zavala, M. Vogelsberger and M.G. Walker, Constraining Self-Interacting Dark Matter with the Milky Way's dwarf spheroidals, Mon. Not. Roy. Astron. Soc. 431 (2013) L20 [arXiv: 1211.6426] [INSPIRE].

[12] M. Vogelsberger, J. Zavala, C. Simpson and A. Jenkins, Dwarf galaxies in CDM and SIDM with baryons: observational probes of the nature of dark matter, Mon. Not. Roy. Astron. Soc. 444 (2014) 3684 [arXiv: 1405.5216] [INSPIRE].

[13] O.D. Elbert, J.S. Bullock, S. Garrison-Kimmel, M. Rocha, J. Oñorbe and A.H.G. Peter, Core formation in dwarf haloes with self-interacting dark matter: no fine-tuning necessary, Mon. Not. Roy. Astron. Soc. 453 (2015) 29 [arXiv:1412.1477] [InSPIRE].

[14] M. Kaplinghat, S. Tulin and H.-B. Yu, Dark Matter Halos as Particle Colliders: Unified Solution to Small-Scale Structure Puzzles from Dwarfs to Clusters, Phys. Rev. Lett. 116 (2016) 041302 [arXiv: 1508.03339] [INSPIRE].

[15] N. Bernal, X. Chu, C. Garcia-Cely, T. Hambye and B. Zaldivar, Production Regimes for Self-Interacting Dark Matter, JCAP 03 (2016) 018 [arXiv:1510.08063] [INSPIRE].

[16] J.L. Feng, M. Kaplinghat and H.-B. Yu, Halo Shape and Relic Density Exclusions of Sommerfeld-Enhanced Dark Matter Explanations of Cosmic Ray Excesses, Phys. Rev. Lett. 104 (2010) 151301 [arXiv:0911.0422] [INSPIRE].

[17] M.R. Buckley and P.J. Fox, Dark Matter Self-Interactions and Light Force Carriers, Phys. Rev. D 81 (2010) 083522 [arXiv:0911.3898] [INSPIRE].

[18] A. Loeb and N. Weiner, Cores in Dwarf Galaxies from Dark Matter with a Yukawa Potential, Phys. Rev. Lett. 106 (2011) 171302 [arXiv:1011.6374] [INSPIRE].

[19] S. Tulin, H.-B. Yu and K.M. Zurek, Beyond Collisionless Dark Matter: Particle Physics Dynamics for Dark Matter Halo Structure, Phys. Rev. D 87 (2013) 115007 [arXiv: 1302.3898] [INSPIRE].

[20] E.D. Carlson, M.E. Machacek and L.J. Hall, Self-interacting dark matter, Astrophys. J. 398 (1992) 43 [INSPIRE].

[21] Y. Hochberg, E. Kuflik, T. Volansky and J.G. Wacker, Mechanism for Thermal Relic Dark Matter of Strongly Interacting Massive Particles, Phys. Rev. Lett. 113 (2014) 171301 [arXiv: 1402.5143] [INSPIRE].

[22] N. Bernal and X. Chu, $\mathbb{Z}_{2}$ SIMP Dark Matter, JCAP 01 (2016) 006 [arXiv:1510.08527] [INSPIRE].

[23] M. Kaplinghat, S. Tulin and H.-B. Yu, Direct Detection Portals for Self-interacting Dark Matter, Phys. Rev. D 89 (2014) 035009 [arXiv:1310.7945] [INSPIRE]. 
[24] N. Bernal, C. Garcia-Cely and R. Rosenfeld, WIMP and SIMP Dark Matter from the Spontaneous Breaking of a Global Group, JCAP 04 (2015) 012 [arXiv:1501.01973] [INSPIRE].

[25] H.M. Lee and M.-S. Seo, Communication with SIMP dark mesons via Z'-portal, Phys. Lett. B 748 (2015) 316 [arXiv: 1504.00745] [InSPIRE].

[26] G. Feinberg and J. Sucher, Long-Range Forces from Neutrino-Pair Exchange, Phys. Rev. 166 (1968) 1638 [INSPIRE].

[27] S.D. McDermott, H.-B. Yu and K.M. Zurek, Turning off the Lights: How Dark is Dark Matter?, Phys. Rev. D 83 (2011) 063509 [arXiv:1011.2907] [INSPIRE].

[28] Q.-H. Cao, E. Ma and G. Rajasekaran, Observing the Dark Scalar Doublet and its Impact on the Standard-Model Higgs Boson at Colliders, Phys. Rev. D 76 (2007) 095011 [arXiv:0708.2939] [INSPIRE].

[29] M. Garny, A. Ibarra and S. Vogl, Signatures of Majorana dark matter with t-channel mediators, Int. J. Mod. Phys. D 24 (2015) 1530019 [arXiv:1503.01500] [InSPIRE].

[30] M. Lindner, A. Merle and V. Niro, Enhancing Dark Matter Annihilation into Neutrinos, Phys. Rev. D 82 (2010) 123529 [arXiv:1005.3116] [InSPIRE].

[31] B. Patt and F. Wilczek, Higgs-field portal into hidden sectors, hep-ph/0605188 [INSPIRE].

[32] R. Essig et al., Working Group Report: New Light Weakly Coupled Particles, in proceedings of the Community Summer Study 2013: Snowmass on the Mississippi. (CSS2013), Minneapolis, MN, U.S.A., July 29-August 62013 [arXiv:1311.0029] [INSPIRE].

[33] J.-H. Huh, J.E. Kim, J.-C. Park and S.C. Park, Galactic 511 keV line from MeV milli-charged dark matter, Phys. Rev. D 77 (2008) 123503 [arXiv:0711.3528] [INSPIRE].

[34] M. Pospelov, A. Ritz and M.B. Voloshin, Secluded WIMP Dark Matter, Phys. Lett. B 662 (2008) 53 [arXiv:0711.4866] [INSPIRE].

[35] G. Bertone, D. Hooper and J. Silk, Particle dark matter: Evidence, candidates and constraints, Phys. Rept. 405 (2005) 279 [hep-ph/0404175] [INSPIRE].

[36] L. Lopez-Honorez, O. Mena, S. Palomares-Ruiz and A.C. Vincent, Constraints on dark matter annihilation from CMB observationsbefore Planck, JCAP 07 (2013) 046 [arXiv: 1303.5094] [INSPIRE].

[37] G. Steigman, CMB Constraints On The Thermal WIMP Mass And Annihilation Cross Section, Phys. Rev. D 91 (2015) 083538 [arXiv:1502.01884] [INSPIRE].

[38] T.R. Slatyer, Indirect dark matter signatures in the cosmic dark ages. I. Generalizing the bound on s-wave dark matter annihilation from Planck results, Phys. Rev. D 93 (2016) 023527 [arXiv: 1506.03811] [INSPIRE].

[39] B. Henning and H. Murayama, Constraints on Light Dark Matter from Big Bang Nucleosynthesis, arXiv:1205.6479 [INSPIRE].

[40] K. Jedamzik and M. Pospelov, Big Bang Nucleosynthesis and Particle Dark Matter, New J. Phys. 11 (2009) 105028 [arXiv: 0906.2087] [INSPIRE].

[41] Super-Kamiokande collaboration, K. Frankiewicz, Searching for Dark Matter Annihilation into Neutrinos with Super-Kamiokande, in proceedings of the Meeting of the APS Division of Particles and Fields (DPF 2015), Ann Arbor, Michigan, U.S.A., August 4-8 2015 [arXiv: 1510.07999] [INSPIRE]. 
[42] H. Goldberg, Constraint on the Photino Mass from Cosmology, Phys. Rev. Lett. 50 (1983) 1419 [Erratum ibid. 103 (2009) 099905] [INSPIRE].

[43] L. Bergstrom, T. Bringmann, M. Eriksson and M. Gustafsson, Gamma rays from Kaluza-Klein dark matter, Phys. Rev. Lett. 94 (2005) 131301 [astro-ph/0410359] [INSPIRE].

[44] T. Bringmann, L. Bergstrom and J. Edsjo, New Gamma-Ray Contributions to Supersymmetric Dark Matter Annihilation, JHEP 01 (2008) 049 [arXiv:0710.3169] [INSPIRE].

[45] V. Barger, Y. Gao, W.Y. Keung and D. Marfatia, Generic dark matter signature for gamma-ray telescopes, Phys. Rev. D 80 (2009) 063537 [arXiv:0906.3009] [InSPIRE].

[46] F. Giacchino, L. Lopez-Honorez and M.H.G. Tytgat, Scalar Dark Matter Models with Significant Internal Bremsstrahlung, JCAP 10 (2013) 025 [arXiv:1307.6480] [INSPIRE].

[47] K. Griest and D. Seckel, Three exceptions in the calculation of relic abundances, Phys. Rev. D 43 (1991) 3191 [INSPIRE].

[48] Planck collaboration, P.A.R. Ade et al., Planck 2013 results. XVI. Cosmological parameters, Astron. Astrophys. 571 (2014) A16 [arXiv:1303.5076] [INSPIRE].

[49] K.K. Boddy and J. Kumar, Indirect Detection of Dark Matter Using MeV-Range Gamma-Ray Telescopes, Phys. Rev. D 92 (2015) 023533 [arXiv: 1504.04024] [InSPIRE].

[50] P.J. Fox, R. Harnik, J. Kopp and Y. Tsai, LEP Shines Light on Dark Matter, Phys. Rev. D 84 (2011) 014028 [arXiv: 1103.0240] [INSPIRE].

[51] N.F. Bell, Y. Cai and R.K. Leane, Mono-W Dark Matter Signals at the LHC: Simplified Model Analysis, JCAP 01 (2016) 051 [arXiv: 1512.00476] [INSPIRE].

[52] E. Izaguirre, G. Krnjaic, P. Schuster and N. Toro, Analyzing the Discovery Potential for Light Dark Matter, Phys. Rev. Lett. 115 (2015) 251301 [arXiv:1505.00011] [InSPIRE].

[53] E. Izaguirre, G. Krnjaic and M. Pospelov, MeV-Scale Dark Matter Deep Underground, Phys. Rev. D 92 (2015) 095014 [arXiv: 1507.02681] [INSPIRE].

[54] A. Alves, A. Berlin, S. Profumo and F.S. Queiroz, Dark Matter Complementarity and the $Z^{\prime}$ Portal, Phys. Rev. D 92 (2015) 083004 [arXiv:1501.03490] [InSPIRE].

[55] UA2 collaboration, J. Alitti et al., A Search for new intermediate vector mesons and excited quarks decaying to two jets at the CERN pp collider, Nucl. Phys. B 400 (1993) 3 [INSPIRE].

[56] J. Erler, P. Langacker, S. Munir and E. Rojas, Improved Constraints on $Z^{\prime}$ Bosons from Electroweak Precision Data, JHEP 08 (2009) 017 [arXiv:0906.2435] [INSPIRE].

[57] A. Alves, S. Profumo and F.S. Queiroz, The dark $Z^{\prime}$ portal: direct, indirect and collider searches, JHEP 04 (2014) 063 [arXiv:1312.5281] [INSPIRE].

[58] K.S. Babu, C.F. Kolda and J. March-Russell, Implications of generalized $Z-Z^{\prime}$ mixing, Phys. Rev. D 57 (1998) 6788 [hep-ph/9710441] [INSPIRE].

[59] D. Clowe, A. Gonzalez and M. Markevitch, Weak lensing mass reconstruction of the interacting cluster 1E0657-558: Direct evidence for the existence of dark matter, Astrophys. J. 604 (2004) 596 [astro-ph/0312273] [INSPIRE].

[60] M. Markevitch et al., Direct constraints on the dark matter self-interaction cross-section from the merging galaxy cluster 1E065\%-56, Astrophys. J. 606 (2004) 819 [astro-ph/0309303] [INSPIRE]. 
[61] S.W. Randall, M. Markevitch, D. Clowe, A.H. Gonzalez and M. Bradač, Constraints on the Self-Interaction Cross-Section of Dark Matter from Numerical Simulations of the Merging Galaxy Cluster 1E 0657-56, Astrophys. J. 679 (2008) 1173 [arXiv:0704.0261] [INSPIRE].

[62] A. Robertson, R. Massey and V. Eke, What does the Bullet Cluster tell us about Self-Interacting Dark Matter?, arXiv:1605.04307 [INSPIRE].

[63] R.T. D'Agnolo and J.T. Ruderman, Light Dark Matter from Forbidden Channels, Phys. Rev. Lett. 115 (2015) 061301 [arXiv: 1505.07107] [INSPIRE].

[64] BABAR collaboration, B. Aubert et al., Search for Invisible Decays of a Light Scalar in Radiative Transitions $\Upsilon(3 S) \rightarrow \gamma A^{0}$, in proceedings of the 34th International Conference on High Energy Physics (ICHEP 2008), Philadelphia, Pennsylvania, U.S.A., July 30-August 5 2008 [arXiv: 0808.0017] [INSPIRE] and online pdf version at http://www-public.slac.stanford.edu/sciDoc/docMeta.aspx?slacPubNumber=slac-pub-13328.

[65] R. Essig, J. Mardon, M. Papucci, T. Volansky and Y.-M. Zhong, Constraining Light Dark Matter with Low-Energy $e^{+} e^{-}$Colliders, JHEP 11 (2013) 167 [arXiv:1309.5084] [INSPIRE].

[66] BeLLe-II collaboration, B. Wang, The Belle II Experiment and SuperKEKB Upgrade, in proceedings of the 10th International Workshop on $e^{+} e^{-}$collisions from $\Phi$ to $\Psi$ (PHIPSI15), Hefei, Anhui, China, September 23-26 2015 [arXiv:1511.09434] [INSPIRE].

[67] S.N. Gninenko, N.V. Krasnikov, M.M. Kirsanov and D.V. Kirpichnikov, Missing energy signature from invisible decays of dark photons at the CERN SPS, arXiv:1604.08432 [INSPIRE].

[68] B. Batell, M. Pospelov and A. Ritz, Exploring Portals to a Hidden Sector Through Fixed Targets, Phys. Rev. D 80 (2009) 095024 [arXiv: 0906.5614] [InSPIRE].

[69] S. Alekhin et al., A facility to Search for Hidden Particles at the CERN SPS: the SHiP physics case, Rept. Prog. Phys. 79 (2016) 124201 [arXiv: 1504.04855] [INSPIRE].

[70] B. Batell, R. Essig and Z. Surujon, Strong Constraints on Sub-GeV Dark Sectors from SLAC Beam Dump E137, Phys. Rev. Lett. 113 (2014) 171802 [arXiv:1406.2698] [InSPIRE].

[71] D. Curtin, R. Essig, S. Gori and J. Shelton, Illuminating Dark Photons with High-Energy Colliders, JHEP 02 (2015) 157 [arXiv:1412.0018] [INSPIRE].

[72] R. Barbieri and T.E.O. Ericson, Evidence Against the Existence of a Low Mass Scalar Boson from Neutron-Nucleus Scattering, Phys. Lett. B 57 (1975) 270 [INSPIRE].

[73] NA48/2 collaboration, J.R. Batley et al., Search for the dark photon in $\pi^{0}$ decays, Phys. Lett. B 746 (2015) 178 [arXiv: 1504.00607] [INSPIRE].

[74] J.L. Feng et al., Protophobic Fifth-Force Interpretation of the Observed Anomaly in ${ }^{8}$ Be Nuclear Transitions, Phys. Rev. Lett. 117 (2016) 071803 [arXiv: 1604.07411] [INSPIRE].

[75] HPS collaboration, A. Celentano, The Heavy Photon Search experiment at Jefferson Laboratory, J. Phys. Conf. Ser. 556 (2014) 012064 [arXiv: 1505. 02025] [InSPIRE].

[76] P. Ilten, J. Thaler, M. Williams and W. Xue, Dark photons from charm mesons at LHCb, Phys. Rev. D 92 (2015) 115017 [arXiv: 1509.06765] [INSPIRE].

[77] N. Fornengo, C.W. Kim and J. Song, Finite temperature effects on the neutrino decoupling in the early universe, Phys. Rev. D 56 (1997) 5123 [hep-ph/9702324] [INSPIRE].

[78] J.H. Heo and C.S. Kim, Light Dark Matter and Dark Radiation, J. Korean Phys. Soc. 68 (2016) 715 [arXiv:1504.00773] [INSPIRE]. 
[79] Topical Conveners: K.N. Abazajian, J.E. Carlstrom, A.T. Lee collaboration, K.N. Abazajian et al., Neutrino Physics from the Cosmic Microwave Background and Large Scale Structure, Astropart. Phys. 63 (2015) 66 [arXiv:1309.5383] [INSPIRE].

[80] A. Manzotti, S. Dodelson and Y. Park, External priors for the next generation of CMB experiments, Phys. Rev. D 93 (2016) 063009 [arXiv:1512.02654] [INSPIRE].

[81] A. Fradette, M. Pospelov, J. Pradler and A. Ritz, Cosmological Constraints on Very Dark Photons, Phys. Rev. D 90 (2014) 035022 [arXiv: 1407.0993] [InSPIRE].

[82] R. Essig, M. Fernandez-Serra, J. Mardon, A. Soto, T. Volansky and T.-T. Yu, Direct Detection of sub-GeV Dark Matter with Semiconductor Targets, JHEP 05 (2016) 046 [arXiv: 1509.01598] [INSPIRE].

[83] P. Batra, B.A. Dobrescu and D. Spivak, Anomaly-free sets of fermions, J. Math. Phys. 47 (2006) 082301 [hep-ph/0510181] [INSPIRE].

[84] R. Essig, J. Mardon and T. Volansky, Direct Detection of Sub-GeV Dark Matter, Phys. Rev. D 85 (2012) 076007 [arXiv: 1108.5383] [INSPIRE].

[85] R. Essig, A. Manalaysay, J. Mardon, P. Sorensen and T. Volansky, First Direct Detection Limits on sub-GeV Dark Matter from XENON10, Phys. Rev. Lett. 109 (2012) 021301 [arXiv: 1206.2644] [INSPIRE]. 